\title{
Interactions of Single-Nozzle Sonic Propulsive Deceleration Jets on Mars Entry Aeroshells
}

\author{
Hicham Alkandry* and Iain D. Boyd ${ }^{\dagger}$ \\ Department of Aerospace Engineering, University of Michigan, Ann Arbor, MI, 48109 \\ Erin M. Reed ${ }^{\ddagger}$, Joshua R. Codoni ${ }^{\S}$, and James C. McDaniel ${ }^{\llbracket}$ \\ Department of Mechanical and Aerospace Engineering, University of Virginia, Charlottesville, VA, 22903
}

\begin{abstract}
Due to scientific interest in increasing the mass of Mars entry systems and the altitude of their landing sites, the size requirements for the conventional aerodynamic decelerators used to slow the vehicle from hypersonic velocities in the upper atmosphere to zero velocity on the surface may become unfeasible. One option is propulsive decelerator (PD) jets which may be used to slow the vehicle down to appropriate speeds. The use of these PD jets, however, involve complex flow interactions that are still not well understood. This paper describes numerical and experimental techniques that are used in an effort to understand these interactions. The numerical simulations use a scaled version of the Mars Science Laboratory aeroshell geometry with a sonic PD jet in a single-nozzle configuration located at the center of the forebody in Mach 12 flow of iodine-seeded nitrogen gas. The boundary conditions for the PD jet are non-dimensionalized using the thrust coefficient in order to compare the numerical results with experimental data from previous and ongoing work. The results show that the flowfield features, such as the bow shock, PD jet shock, and recirculation regions in front the aeroshell's forebody, are all affected by the thrust coefficient of the PD nozzle. These effects also extend to the surface and aerodynamic properties of the aeroshell. The results show that as the thrust coefficient increases, the absolute values of the pressure and shear stress along the surface of the aeroshell decrease and approach a roughly constant value over the entire surface. The aerodynamic drag coefficient of the aeroshell also decreases and approaches an almost constant value equal to $8 \%$ of the value for the PD jet off case as the thrust coefficient increases. Finally, comparisons between the numerical results and experimental data show good agreement in the bow shock profile and standoff distance as well as the aerodynamic properties of the aeroshell between the two sets of results.
\end{abstract}

\section{Nomenclature}

$\begin{array}{ll}\mathrm{M} & \text { Mach Number } \\ \mathrm{P} & \text { Static Pressure }[\mathrm{Pa}] \\ \mathrm{q} & \text { Dynamic Pressure }[\mathrm{Pa}] \\ \tau & \text { Shear Stress }[\mathrm{Pa}] \\ \mathrm{D} & \text { Drag Force }[\mathrm{N}] \\ \mathrm{T} & \text { Thrust Force }[\mathrm{N}] \\ \rho & \text { Density }\left[\mathrm{kg} / \mathrm{m}^{3}\right] \\ \mathrm{U} & \text { Velocity }[\mathrm{m} / \mathrm{s}] \\ \mathrm{S} & \text { Aeroshell Frontal Area }\left[\mathrm{m}^{2}\right] \\ C_{T} & \text { Thrust Coefficient }\end{array}$

\footnotetext{
* Graduate Student, Student Member AIAA.

†Professor, Associate Fellow AIAA.

$\ddagger_{\text {Graduate Student, Student Member AIAA. }}$

$\S$ Graduate Student, Student Member AIAA.

"Professor, Senior Member AIAA.
} 


$\begin{array}{ll}C_{P} & \text { Pressure Coefficient } \\ C_{f} & \text { Coefficient of Skin Friction } \\ C_{D} & \text { Aerodynamic Drag Coefficient } \\ & \\ \text { subscripts } \\ \infty & \text { Freestream } \\ \text { jet } & \text { PD Jet } \\ 0 & \text { Total (Stagnation) }\end{array}$

\section{Introduction}

Conventional aerodynamic decelerators for future Mars landers may be insufficient due to extremely large parachute size requirements. The Mars Science Laboratory (MSL) spacecraft, scheduled for launch in the Fall of 2011, has an estimated landing mass larger than $1700 \mathrm{~kg}$, which is far greater than the entry mass for any previous Mars entry system (e.g. Viking). ${ }^{1}$ The MSL will also land at a site that is up to $1 \mathrm{~km}$ above the reference altitude. Future missions, including possible human missions, may continue this trend of carrying more payload masses to Mars in order to conduct more sophisticated in situ experiments and landing at sites of scientific interest that are at higher altitudes. However, it may not be possible to simply extend the Viking-heritage technology (e.g. supersonic Disk-Gap-Band parachutes and $70^{\circ}$ blunt body aeroshells) to the dimensions and deployment conditions required by these missions. ${ }^{2}$ These challenges may be resolved by using an additional propulsive decelerator (PD) component in order to slow the vehicle down to appropriate speeds.

Previous work on PD jets was conducted in the 1960s and early 1970s primarily through wind tunnel experiments. This work mainly examined the aerodynamic effects of the PD jets for both single- and multiplenozzle configurations, which are shown in Figs.1(a) and 1(b), respectively. Experimental results showed that for relatively low non-dimensional nozzle thrust values, only a small augmentation of the axial force (the sum of the aerodynamic drag and the thrust forces) beyond that provided by the PD jet off case was observed for the single-nozzle configuration. ${ }^{3}$ Beyond the 1970s, however, there has not been any significant work on propulsive deceleration and several important limitations still exist. These limitations include a lack of extensive experimental data and validated numerical approaches that can accurately and efficiently simulate the complex flow interactions that are generated in the use of these PD jets.

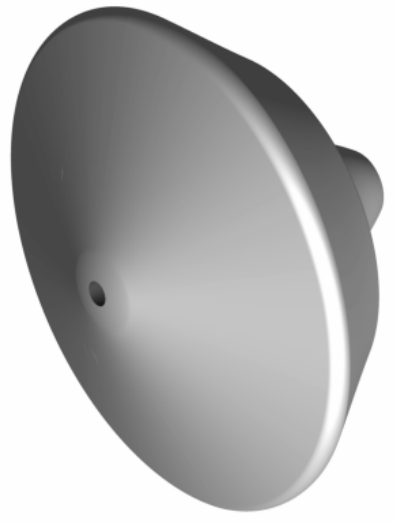

(a) Single-nozzle.

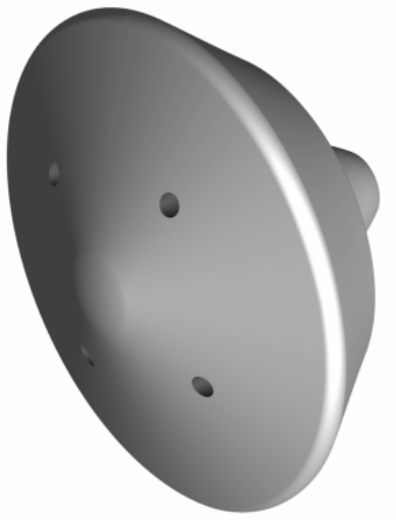

(b) Multiple-nozzles.

Figure 1. PD jet nozzle configurations.

This paper will describe numerical and experimental approaches that are used to understand the complex flow interactions between the PD jets, the freestream, and the aeroshell. It will also present numerical results using a scaled version of the MSL aeroshell geometry with a sonic PD jet in a single-nozzle configuration located at the center of the forebody. These results are presented in four parts. The first, second, and third sections of this paper will focus on the effects of the PD jet on the flowfield, surface, and aerodynamic properties of the aeroshell, respectively. In the last section of the paper, comparisons between numerical and 
experimental results will be presented to assess the computational method.

\section{Technical Approach}

\section{A. Experimental Technique}

Experimental measurements are obtained using the planar laser-induced iodine fluorescence (PLIIF) technique at a hypersonic wind tunnel facility at the University of Virginia. The PLIIF technique is a nonintrusive, spatially-resolved, time-averaged optical method for measurements in hypersonic, rarefied flows. The technique has been used for both qualitative and quantitative measurements. ${ }^{4-6}$ PLIIF involves seeding iodine into a flowfield and exciting the molecules to a higher energy with an argon ion laser. The laser beam is turned into a thin laser sheet and passed through the flowfield of interest. The resulting fluorescence is imaged at 90 degrees using a cooled scientific-grade charge-coupled device (CCD) camera. Measurements of the absorption spectrum are made as the laser is tuned in frequency. By fitting the measured absorption spectra at every point in the flowfield, the velocity, temperature and injectant mole fraction can be deduced.

The hypersonic flow facility at the University of Virginia is capable of providing Mach numbers and Knudsen numbers up to 16 and 1, respectively. Hypersonic flow from an under-expanded jet is produced by the expansion of iodine-seeded nitrogen gas across a thin circular orifice of diameter $D=2 \mathrm{~mm}$ into a continuously evacuated vacuum chamber. The stagnation pressure and temperature in the wind tunnel are $1.8 \mathrm{~atm}$ and $300 \mathrm{~K}$, respectively. Figure 2(a) presents a schematic of the experimental setup in the hypersonic flow facility. Figure 2(b) shows calculated Mach number and Knudsen number $(K n)$ variations inside the freejet facility. ${ }^{5}$ These contours show the barrel shock that develops at the entrance of the test section and terminates at the Mach disk. Models are placed in the under-expanded jet core for testing at hypersonic conditions. The freestream Mach number and flow properties can be changed by adjusting the distance of the test model to the orifice.

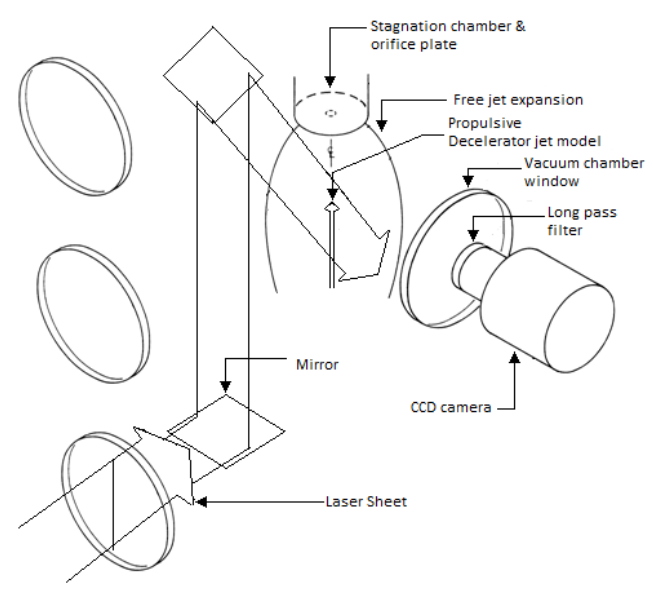

(a) Schematic of the experimental setup.

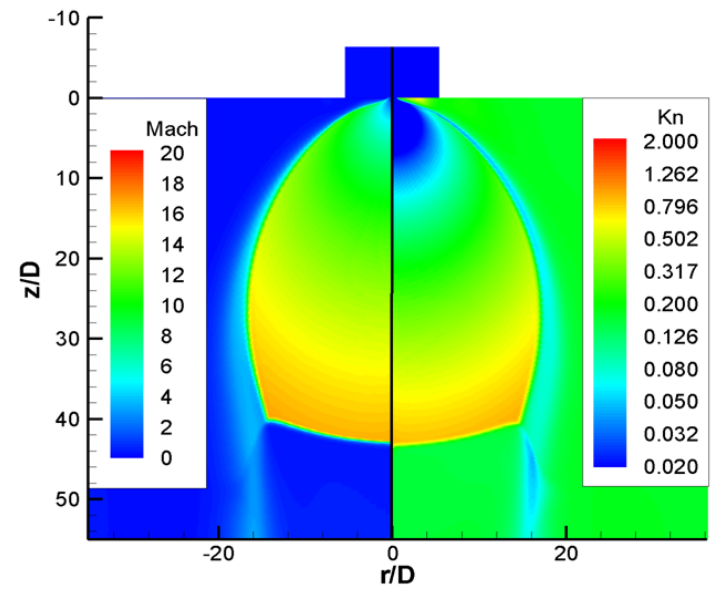

(b) Calculated Mach number and Knudsen number contours in the test section.

Figure 2. Experimental facility.

\section{B. Numerical Method}

Numerical simulations are performed using the computational fluid dynamics (CFD) code LeMANS, developed at the University of Michigan for simulating hypersonic reacting flows. ${ }^{7-9}$ This general purpose, three-dimensional, parallel code solves the laminar Navier-Stokes equations on unstructured computational grids including thermo-chemical nonequilibrium effects with second-order accuracy. The flow is modeled assuming that the continuum approximation is valid. Furthermore, it is assumed that the translational and rotational energy modes of all species can be described by two different temperatures $T_{\text {tra }}$ and $T_{\text {rot }},{ }^{10}$ 
respectively, while the vibrational energy mode and electron energy of all species can be described by a single temperature $T_{v i b}$. The electronic energy is neglected due to the relatively small temperatures achieved in the hypersonic flows of interest. In LeMANS, the mixture transport properties can be computed using several options. In this study, Wilke's semi-empirical mixing ${ }^{11}$ is used with species viscosities calculated using Blottner's model ${ }^{12}$ and species thermal conductivities determined using Eucken's relation. ${ }^{13}$

The finite-volume method applied to unstructured grids is used to solve the set of differential equations. LeMANS can simulate two-dimensional/axisymmetric flows using any mixture of quadrilateral and triangular mesh cells, and three-dimensional flows using any mixture of hexahedra, tetrahedra, prisms, and pyramids. A modified Steger-Warming Flux Vector Splitting scheme is used to discretize the inviscid fluxes across cell faces, which is less dissipative and produces better results in boundary layers compared to the original scheme. The viscous terms are computed using cell-centered and nodal values. Time integration is performed using either a point implicit or a line implicit method. LeMANS is parallelized using METIS ${ }^{14}$ to partition the computational mesh and MPI to communicate the necessary information between processors.

\section{Numerical Setup}

The geometry of the model used in this study is shown in Fig. 3. The model is $10 \mathrm{~mm}$ in diameter, which is equivalent to approximately $0.22 \%$ the size of the MSL aeroshell. The PD jet is located at the center of the forebody. The PD nozzle consists of a converging section, with a nozzle exit diameter of $0.5 \mathrm{~mm}$ and an inlet-to-exit area ratio equal to 21.

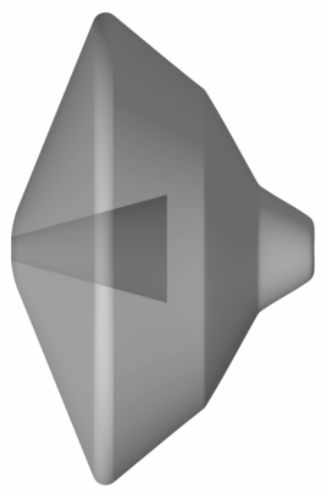

(a) Side view.

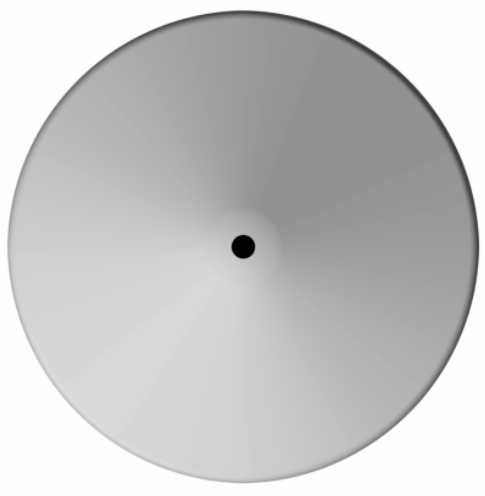

(b) Front view.

Figure 3. Model geometry.

In order to accurately simulate the flow in the experimental facility, $I_{2}$-seeded $N_{2}$ gas is used in the numerical simulations with a seeding ratio of $200 \mathrm{ppm}$. Radially nonuniform conditions based on the freejet relations of Ashkenas and Sherman ${ }^{15}$ are also used as flow conditions input to LeMANS at the upstream boundary. A previous study showed that these nonuniform freestream conditions widen the bow shock around the aeroshell and decrease the drag coefficient by $6.4 \%$ compared to the uniform conditions. ${ }^{16}$ The Mach number at a distance $z$ away from the orifice along the centerline of the freejet is given by Eq. 1

$$
M=A\left(\frac{z-z_{0}}{D}\right)^{\gamma-1}-\frac{1}{2}\left(\frac{\gamma+1}{\gamma-1}\right)\left[A\left(\frac{z-z_{0}}{D}\right)^{\gamma-1}\right]^{-1}
$$

where $D$ is the diameter of the freejet orifice, and $A$ and $z_{0} / D$ are constants determined for values of the ratio of specific heats $\gamma$ and are equal to 3.65 and 0.40 , respectively, for $\gamma=1.4$. All other fluid properties along the freejet axis can be computed using the Mach number defined in Eq. 1, the stagnation conditions in the wind tunnel and the isentropic relations. The density distribution at a fixed distance from the orifice exit is a function of the streamline angle $\theta$ with respect to the freejet axis as shown in Eq. 2

$$
\frac{\rho(\theta)}{\rho(0)}=\cos ^{2}\left(\frac{\pi \theta}{2 \Phi}\right)
$$


where $\Phi$ is also a constant determined for each value of $\gamma$ and is equal to 1.662 for $\gamma=1.4$. For this study, a freestream Mach number of 12 is used in order to minimize the interaction of the bow shock generated by the model and the barrel shock created in the test section in the experiments. As a result, it is not necessary to model the entire test section of the wind tunnel in the numerical simulations, which dramatically cuts down on the computational cost and complexity. Figure 4, modified from Cecil and McDaniel, ${ }^{5}$ shows a to-scale plot of the location of the aeroshell model with respect to the freejet orifice and velocity streamlines for the Ashkenas and Sherman boundary conditions.

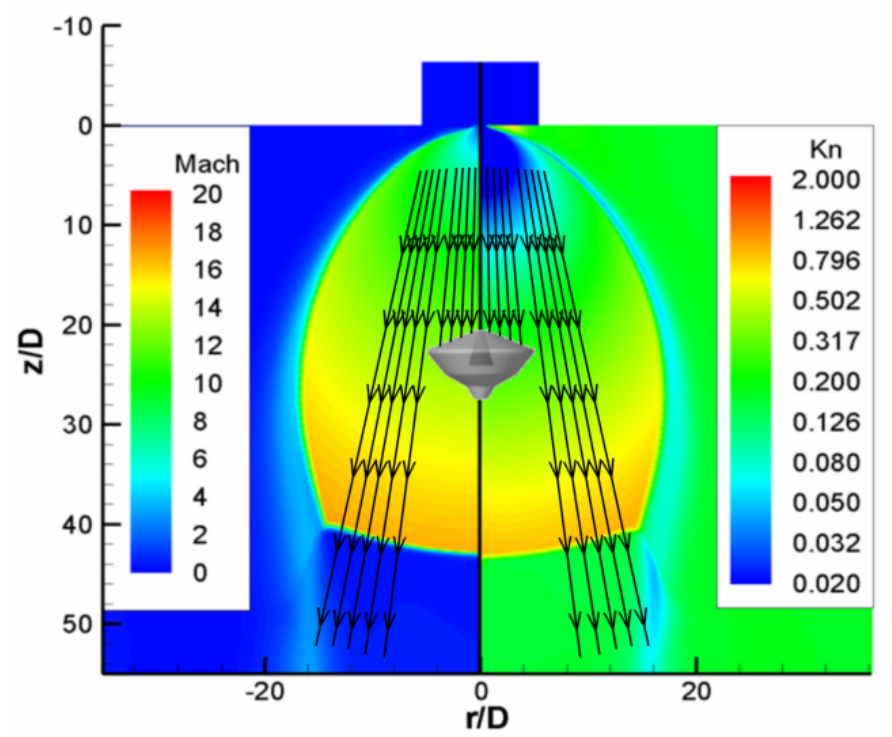

Figure 4. Ashkenas and Sherman boundary conditions and the position of the model in the test section.

The boundary conditions for the PD jet are computed such that sonic conditions are obtained at the nozzle exit. These conditions are non-dimensionalized using the thrust coefficient, as defined by McGhee ${ }^{17}$ in order to compare the results with other previous and ongoing work. ${ }^{6,17}$ The thrust coefficient of a nozzle is defined as the ratio of the thrust produced by the nozzle to the product of the freestream dynamic pressure and the aeroshell frontal area. The expression for the thrust coefficient is given in Eq. 3

$$
C_{T}=\frac{T}{q_{\infty} S}=\left(2 \frac{q_{j e t}}{q_{\infty}}+\frac{P_{j e t}}{q_{\infty}}-\frac{P_{a m b}}{q_{\infty}}\right)\left(\frac{A_{j e t}}{S}\right)
$$

where $T$ is the thrust produced by the nozzle, $q$ is the dynamic pressure, $S$ is the aeroshell frontal area, and $P$ is the static pressure. The ambient static pressure value in Eq. 3 (i.e. $P_{a m b}$ ) is the post-bow-shock pressure value. Table 1 shows the boundary conditions at the nozzle exit for the thrust coefficients that are used in this study.

Table 1. PD jet boundary conditions (at nozzle exit).

\begin{tabular}{|c|c|c|c|}
\hline$C_{T}$ & $P_{0, j} / P_{0}$ & $P_{j}[\mathrm{~Pa}]$ & $\rho_{j}\left[\mathrm{~kg} / \mathrm{m}^{3}\right]$ \\
\hline \hline 0.5 & 0.11 & 10,700 & 0.14 \\
\hline 1.0 & 0.22 & 21,300 & 0.29 \\
\hline 1.5 & 0.33 & 32,100 & 0.43 \\
\hline 2.0 & 0.44 & 42,600 & 0.54 \\
\hline 2.5 & 0.55 & 53,200 & 0.72 \\
\hline
\end{tabular}

Due to the symmetry of the flowfield, axisymmetric simulations are performed using LeMANS in order to reduce the computational cost and complexity of these simulations. Figure 5 shows some of the computational 
grids that are used in this study. These meshes are generated using the commercial software Pointwise ${ }^{18}$ and are adapted by hand from previous simulations to align the upstream boundary of the computational domain with the bow shock. The grids are structured with quadrilateral elements because the numerical results are sensitive to the alignment of the bow shock with the grid. Cells are clustered near the wall and in the vicinity of the PD jet in front of the aeroshell. A grid convergence study is also conducted to ensure that the solutions are grid-independent. The grid size varies from about 85,000 cells for the $C_{T}=0.5$ case to approximately 100,500 cells for the $C_{T}=2.5$ case. The average computational runtime for these simulations is approximately $240 \mathrm{CPU}$-hours.

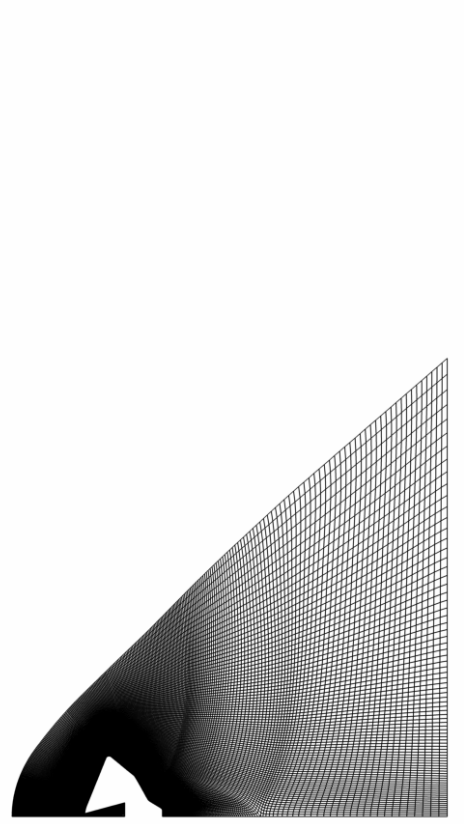

(a) $C_{T}=0.5$

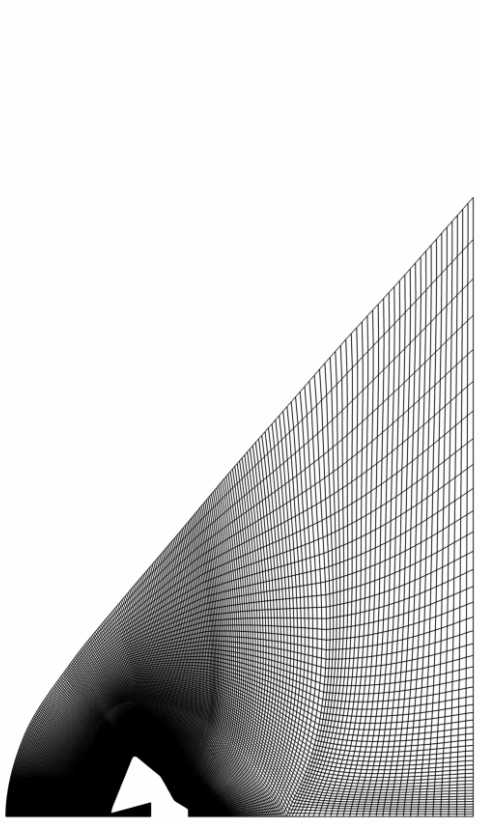

(b) $C_{T}=1.5$

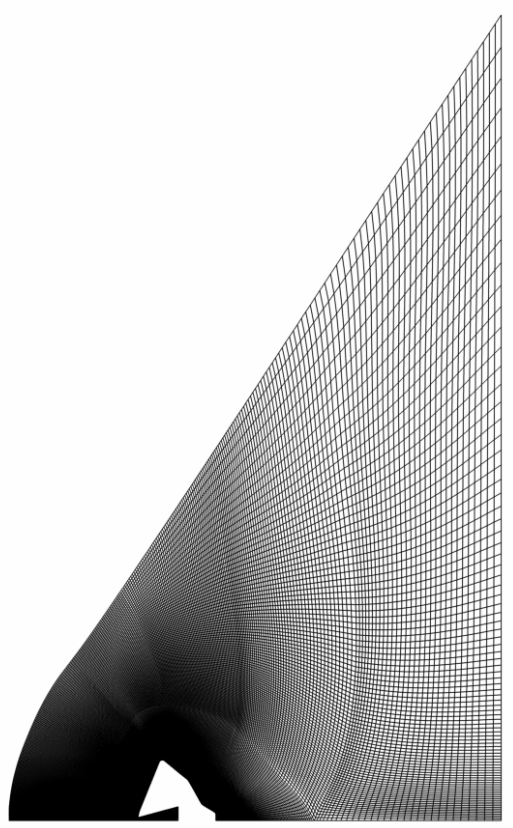

(c) $C_{T}=2.5$

Figure 5. Computational grids.

\section{Results}

The goal of this study is to understand the effects of sonic PD jets in a single-nozzle configuration on the flowfield, surface, and aerodynamic properties of a Mars entry aeroshell using the CFD code LeMANS. The numerical results are also used in comparisons with experimental data from previous and ongoing work to assess the computational method. The flowfield properties that are used to study the effects of these parameters are the Mach number and the PD jet species mole fraction. The surface properties are presented as non-dimensionalized pressure and skin friction coefficients defined by Eqs. 4 and 5, respectively,

$$
\begin{gathered}
C_{P}=\frac{P}{\frac{1}{2} \rho_{\infty} U_{\infty}^{2}} \\
C_{f}=\frac{\tau}{\frac{1}{2} \rho_{\infty} U_{\infty}^{2}}
\end{gathered}
$$

where $P$ is the surface pressure and $\tau$ is the wall shear stress. The drag coefficient, given in Eq. 6 , is used to investigate the aerodynamic effects,

$$
C_{D}=\frac{D}{\frac{1}{2} \rho_{\infty} U_{\infty}^{2} S}
$$

where $D$ is the drag force. 


\section{A. Flowfield Effects}

Figure 6 presents Mach number contours for the 0.5 thrust coefficient conditions. The PD jet expands from sonic conditions at the nozzle exit to higher Mach numbers (i.e. supersonic). The flow then first decelerates from supersonic to subsonic velocities through a jet shock and then from subsonic to zero velocity at a stagnation point detached from the surface of the aeroshell. The freestream also decelerates from hypersonic to subsonic velocities through a bow shock and then to zero velocity at the same stagnation point. In the interface region (region between the bow and jet shocks where the two streams mix), the total pressures for the two streams are equal as they both flow outward between the two shocks with subsequent re-acceleration to supersonic velocities. The figure also shows a region of separated flow between the PD jet boundary, the surface of the model and the mixed outflow, with a reattachment point near the shoulder of the aeroshell.

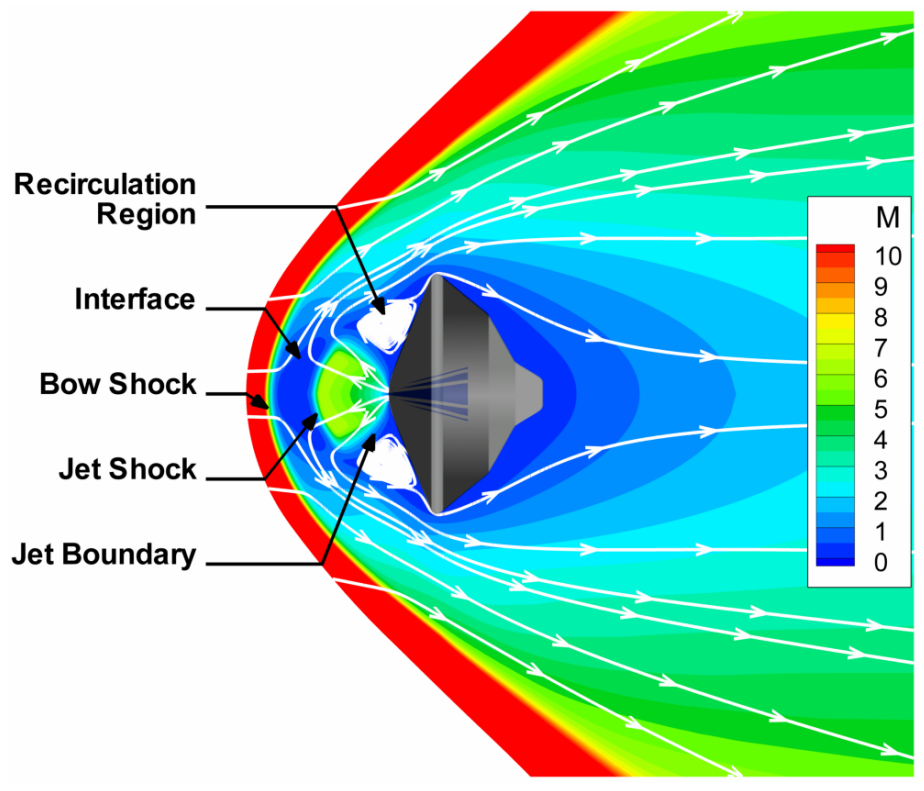

Figure 6. PD jet flowfield features for $C_{T}=\mathbf{0 . 5}$.

Mach number contours for PD nozzle thrust coefficient values of 0.5, 1.0, 2.0, and 2.5 are shown in Fig. 7. The figure shows that the PD jet expands from sonic conditions at the nozzle exit to supersonic conditions for all four thrust coefficient values. The PD jet then decelerates to zero velocity at a detached stagnation point, first through a shock and then subsonically. The figure also shows that all of the flowfield features shown in Fig. 6 are affected by the nozzle thrust coefficient. The bow shock, interface region, and jet shock all move upstream as the thrust coefficient increases in order to equalize the stagnation pressure of the PD jet and the freestream flow. The recirculation regions on either side of the PD jet in front of the aeroshell also decrease in size and move downstream towards the shoulder as the thrust coefficient increases. The reason for this is that the PD jet expands more as the thrust coefficient increases, and therefore, can overcome the relatively sharp turning angle.

Contours of the PD jet mole fraction (i.e. tagged $N_{2}$ species) for nozzle thrust coefficient values of 0.5 , 1.0, 2.0, and 2.5 are presented in Fig. 8. As expected, the size of the PD jet increases with the thrust coefficient. The width of the PD jet grows from approximately half the length of the aeroshell diameter for a thrust coefficient of 0.5 to over a diameter length for a thrust coefficient of 2.5. The amount of PD jet species in the wake also increases as the thrust coefficient increases, since more species are transported downstream by the main flowfield as the mass flow rate of the PD jet increases. This is important because the design of the thermal protection system of the aftbody of the aeroshell will need to consider the hot gases from the PD jet that will be transported downstream of the capsule. 

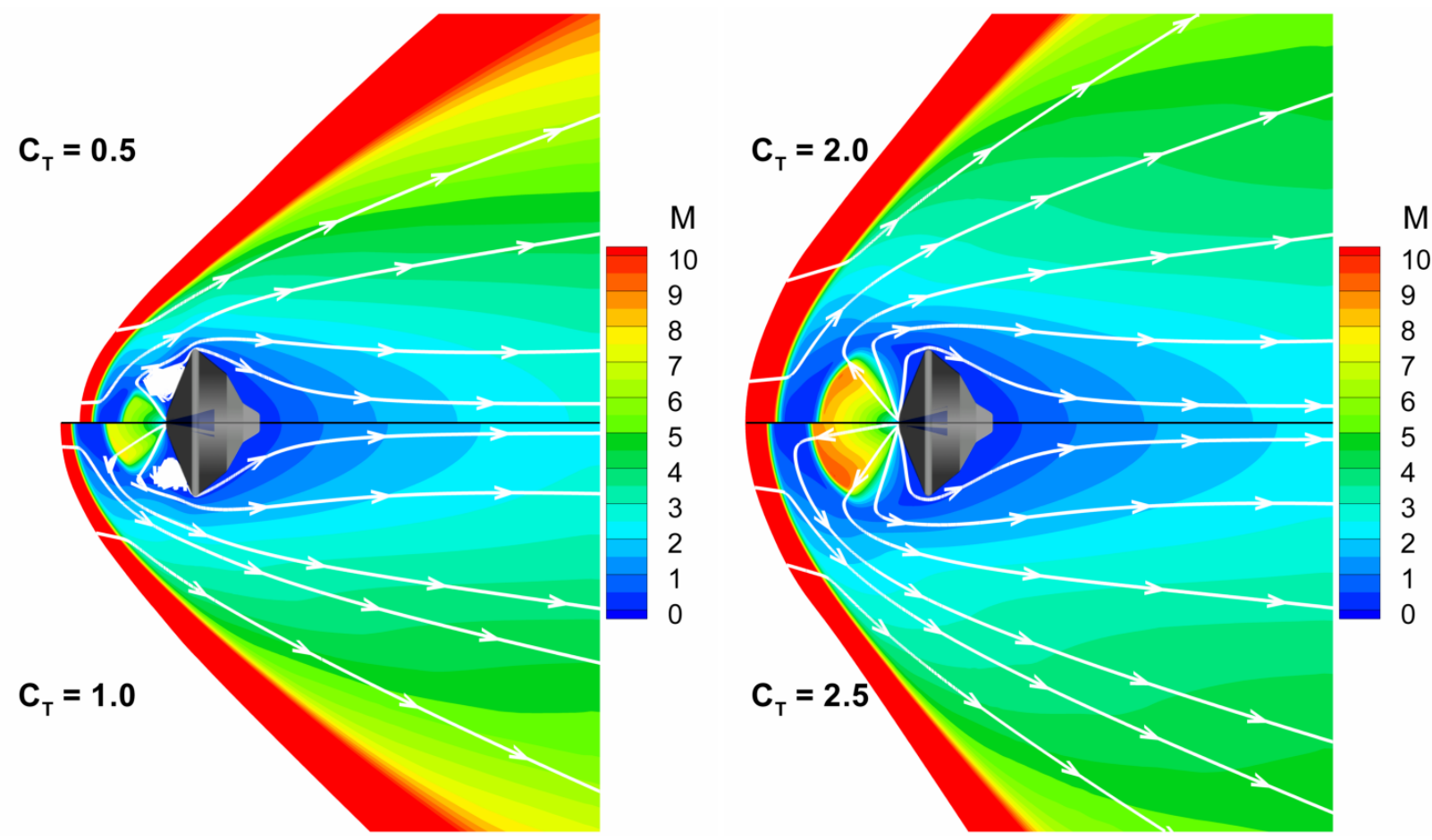

Figure 7. Mach number contours as a function of thrust coefficient.
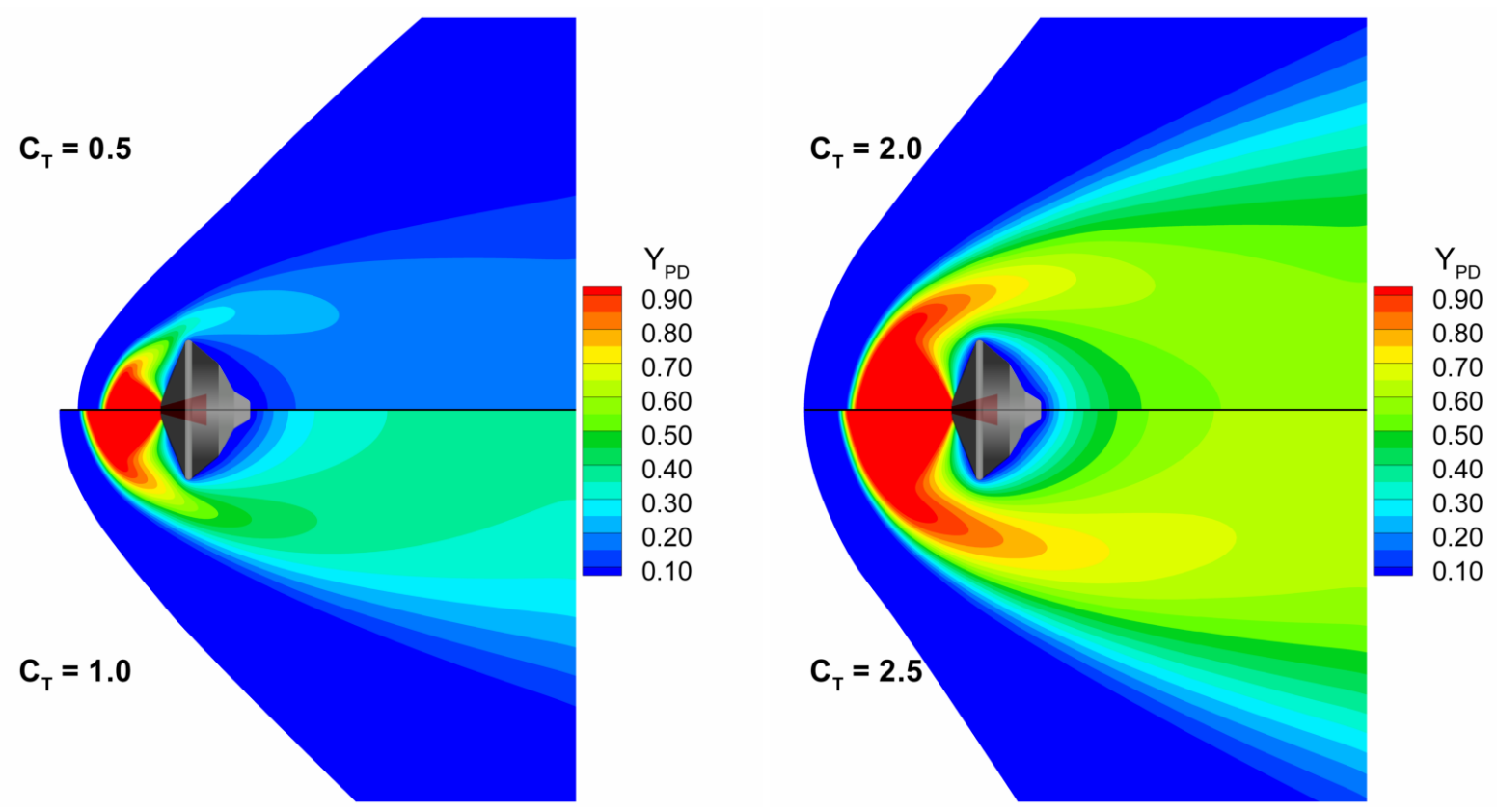

Figure 8. PD jet species mole fraction contours as a function of thrust coefficient. 


\section{B. Surface Effects}

Figure 9 presents the pressure and skin friction coefficients along the surface of the capsule for PD nozzle thrust coefficients of $0.5,1.0,2.0$, and 2.5, as well as for the PD jet off case for comparison. The figure shows that both the surface pressure and shear stress are affected by the thrust coefficient of the PD nozzle. The pressure along the surface first decreases from a high value near the PD jet nozzle-exit. The pressure then increases to a peak near the shoulder of the aeroshell and sharply decreases before finally reaching a roughly constant small value along the aftbody. The magnitude of this peak decreases and the pressure along the forebody approaches an almost constant value equal to the aftbody value as the thrust coefficient increases. The coefficient of skin friction profiles also show that the shear stress along the surface first decreases from a maximum value at the PD jet nozzle-exit. The shear stress then decreases to negative values for thrust coefficients less than approximately 2.0 (i.e. flow reattachment begins near a thrust coefficient of 2.0) and then increases to a larger value at the shoulder before decreasing and finally reaching an almost constant small value along the aftbody of the aeroshell. Similar to the pressure, the overall magnitudes of the coefficient of skin friction decrease and approach a constant value along the entire aeroshell surface as the thrust coefficient increases. The effect of thrust coefficient on the surface properties suggests that the aerodynamic properties of the aeroshell, in particular the drag force, are also affected.

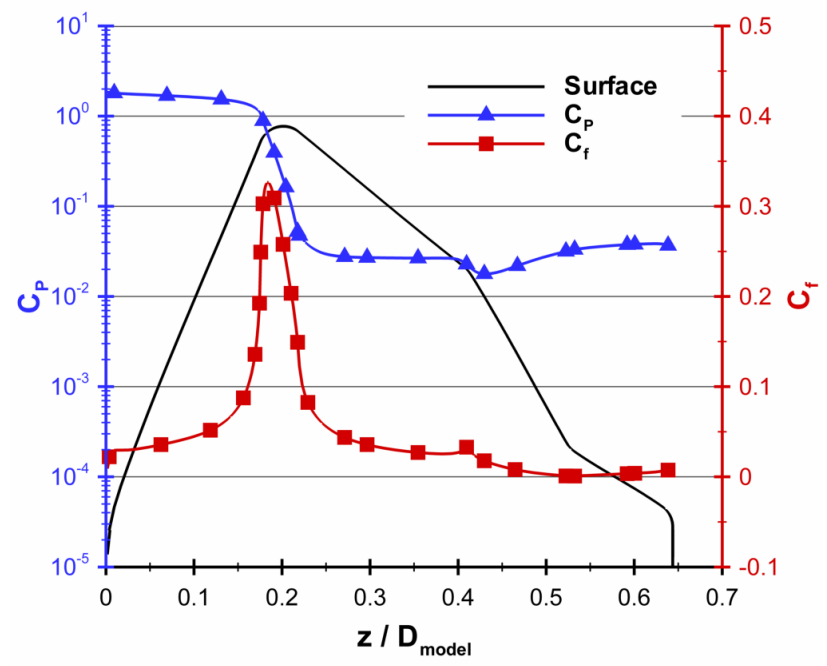

(a) $C_{T}=0.0$

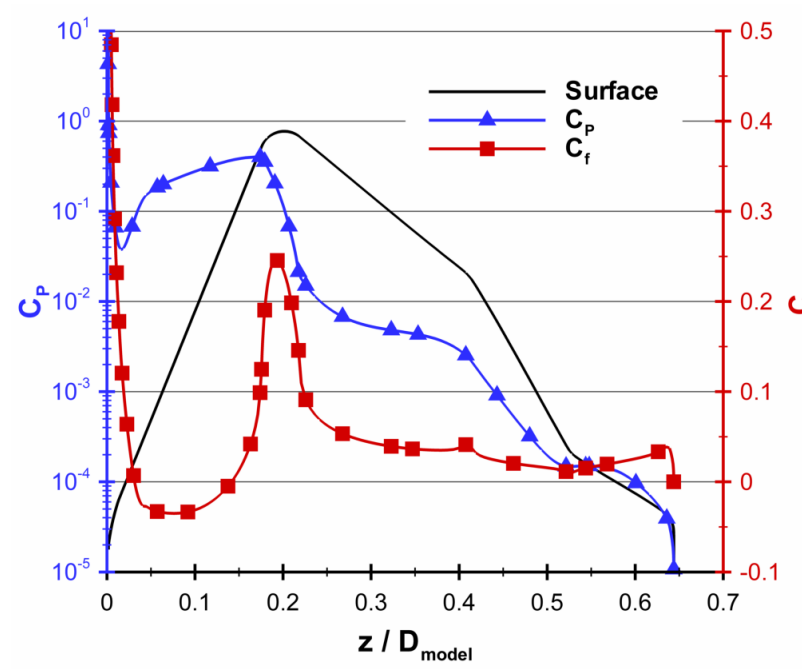

(b) $C_{T}=0.5$

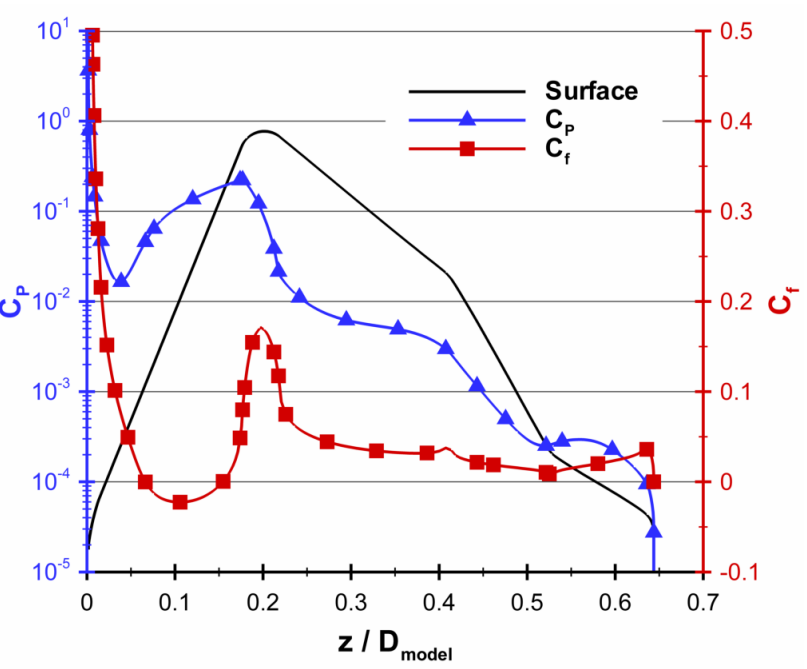

(c) $C_{T}=1.0$

Figure 9. Pressure and skin friction coefficient along the surface of the aeroshell. 


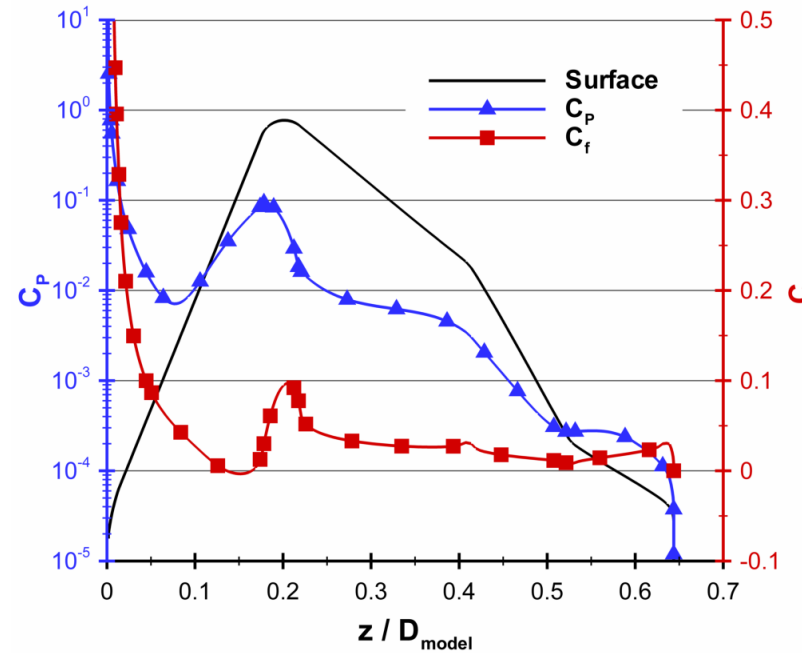

(d) $C_{T}=2.0$

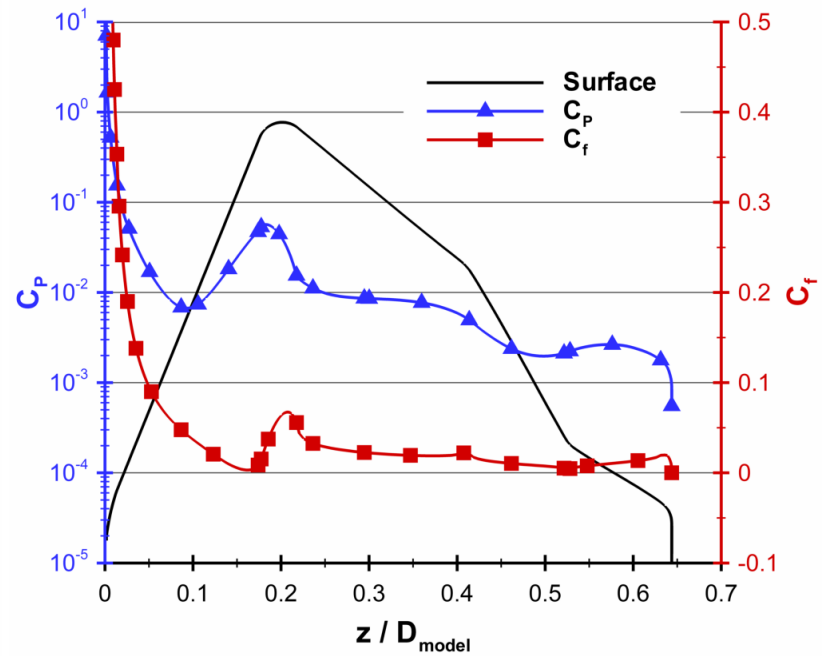

(e) $C_{T}=2.5$

Figure 9. Pressure and skin friction coefficient along the surface of the aeroshell (con't).

The overall decrease in pressure along the surface of the aeroshell with increasing thrust coefficient is caused by a shield effect of the PD jet in the central single-nozzle configuration that prevents mass and momentum from the main freestream flow from reaching the surface of the aeroshell. As the PD jet expands from the nozzle, it pushes the main freestream flow upstream and creates a low pressure region between the jet boundary and the surface. As the thrust coefficient increases, the size of this region also increases since the size of the PD jet and the shield effect also increase. The location of the peak in the pressure profiles near the aeroshell shoulder shown in Fig. 9 corresponds to the point at which the shielding effect of the $\mathrm{PD}$ jet becomes negligible. The magnitude of the peak is roughly equal to the value for the PD jet off case shown in Fig. 9(a). Figure 10 shows mass flux (i.e. $\rho U$ ) and momentum flux (i.e. $P+\rho U^{2}$ ) computed using the density of the main freestream flow (i.e. excluding the PD jet) for thrust coefficient values of 0.5 and 2.5 in order to quantify the amount of mass and momentum transfered to the surface. The figure shows that less mass and momentum from the freestream reach the surface of the aeroshell as the thrust coefficient increases, which decreases the overall surface pressure values.

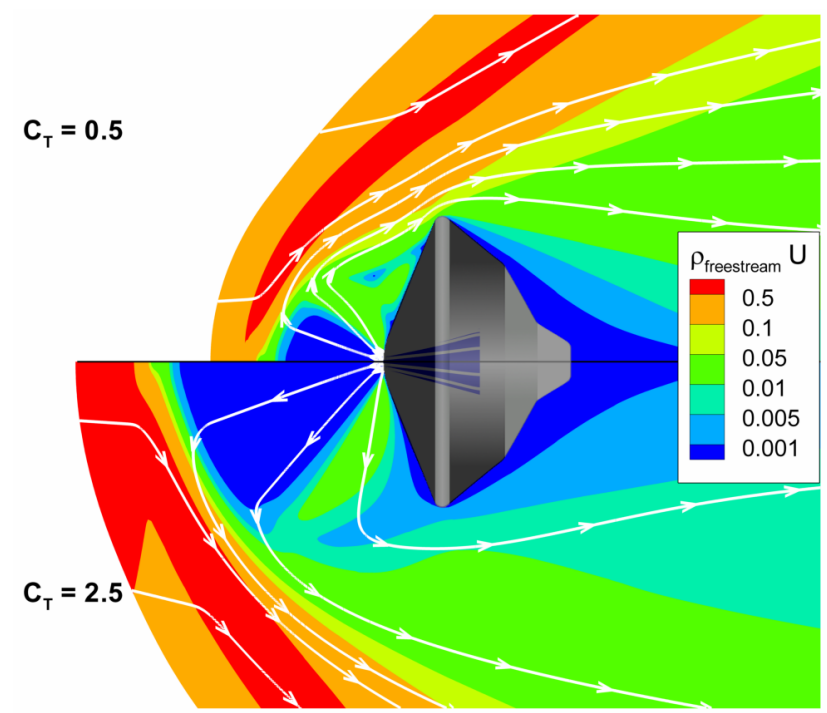

(a) Mass $\left[\mathrm{kg} / \mathrm{s} \cdot \mathrm{m}^{2}\right]$

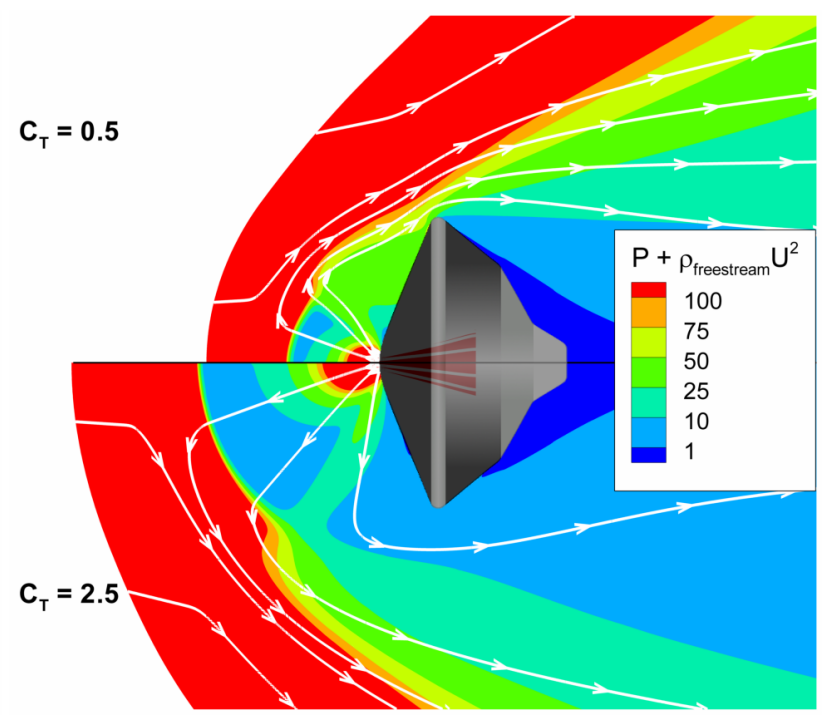

(b) Momentum $\left[\mathrm{N} / \mathrm{m}^{2}\right]$

Figure 10. Mass and momentum transfer from the freestream to the surface of the aeroshell as a function of thrust coefficient. 


\section{Aerodynamic Effects}

Figure 11 presents the aerodynamic drag coefficient, computed using Eq. 6, as a function of thrust coefficient. The drag force is calculated by integrating the pressure and shear stress along the surface of the aeroshell. The figure also presents the total axial force coefficient of the aeroshell, which is equal to the sum of the aerodynamic drag and thrust coefficients. As the thrust coefficient increases, the drag coefficient decreases and asymptotically reaches a constant value that is approximately equal to $8 \%$ of the value for the PD jet off case $\left(C_{D}=1.4\right.$ for $C_{T}=0$ and $C_{D}=0.11$ for $\left.C_{T}=2.5\right)$. The decrease in the aerodynamic drag coefficient with increasing thrust coefficient is due to lower surface pressure along the aeroshell forebody. The figure also shows that the total axial force coefficient first decreases as the thrust coefficient increases, and then begins to increase for thrust coefficient values greater than approximately 0.5 . The total axial force coefficient value does not exceed the drag coefficient value for the PD jet off case until the thrust coefficient is equal to about 1.25 , where most of the contribution to the axial force is from the PD thrust. This suggests that propulsive deceleration using sonic PD jets in a single-nozzle configuration is only beneficial for relatively large thrust coefficient values that are greater than approximately 1.25. The total axial force coefficient increases by roughly constant increments for thrust coefficients greater than 1.25 , since the drag coefficient is almost constant.

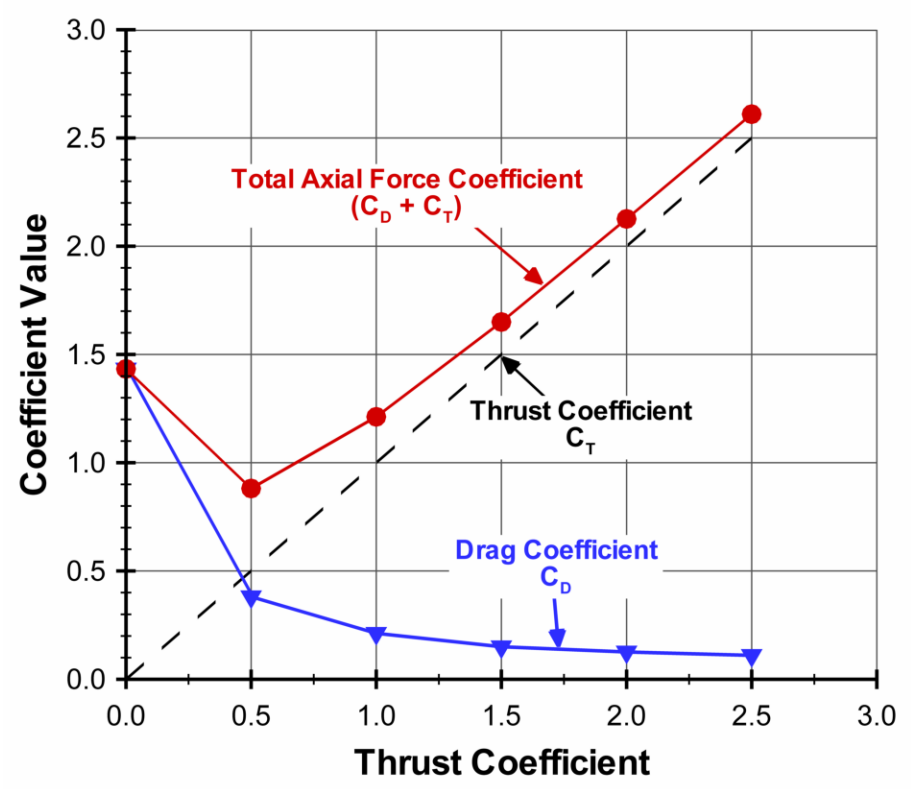

Figure 11. Drag and total axial force coefficients.

\section{Comparison with Experimental Data}

Comparisons between the numerical results and experimental data obtained from previous and current work are carried out to assess the computational method. The images in Fig. 12 are PLIIF visualizations, where the bright areas represent regions with relatively high density values. The lines in the figure are velocity streamlines from the numerical results. The figure shows good qualitative agreement between LeMANS and PLIIF with respect to the bow shock profile around the aeroshell.

Figure 13(a) shows the bow shock standoff distance (i.e. the distance from the center of the aeroshell forebody to the bow shock along the stagnation streamline) for LeMANS and PLIIF. This distance is obtained using the density flowfield and corresponds to the location where the density values begin to increase at the bow shock. As can be seen from the figure, the numerical results from LeMANS are in good agreement with the experimental data over the range of PD jet nozzle thrust coefficient values. The aerodynamic properties from LeMANS are also compared to experimental data obtained by McGhee ${ }^{17}$ in the early 1970s. This experimental work investigated the aerodynamic interactions of supersonic PD jets $\left(M_{\text {jet }}=3.0\right)$ in supersonic flow $\left(M_{\infty}=6.0\right)$ using a $70^{\circ}$ blunt cone geometry with a central single-nozzle 


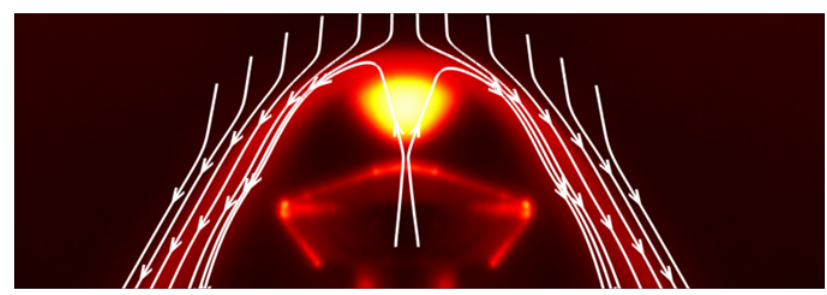

(a) $C_{T}=0.5$

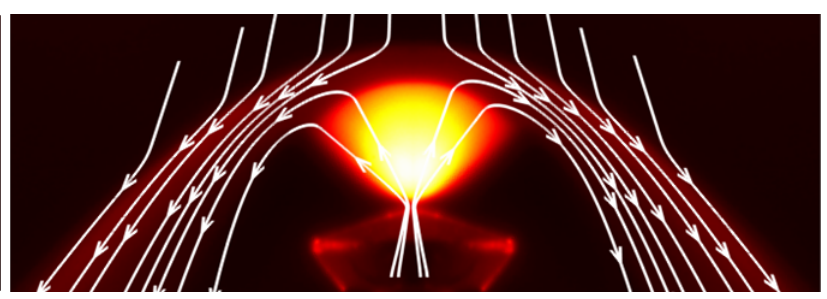

(b) $C_{T}=2.0$

Figure 12. Bow shock profile comparison (images: PLIIF; lines: LeMANS velocity streamlines).

PD jet configuration. Although the freestream and PD jet Mach numbers are different between the present computations and the experimental study, Fig. 13(b) shows overall good agreement between the two sets of results in terms of the aerodynamic effects of single-nozzle PD jets. Both sets of results show similar trends and very close values for the aerodynamic drag and total axial force coefficients.

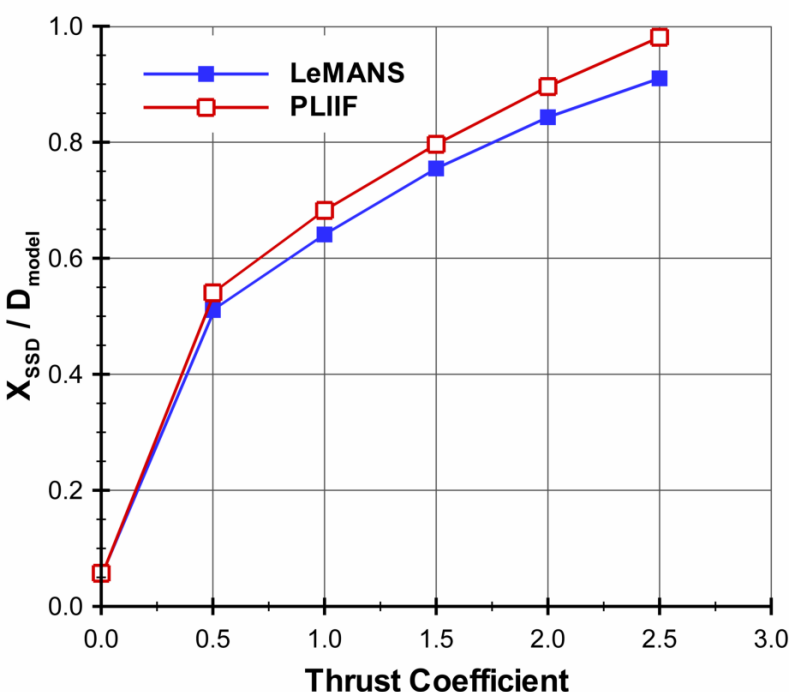

(a) Bow shock standoff distance.

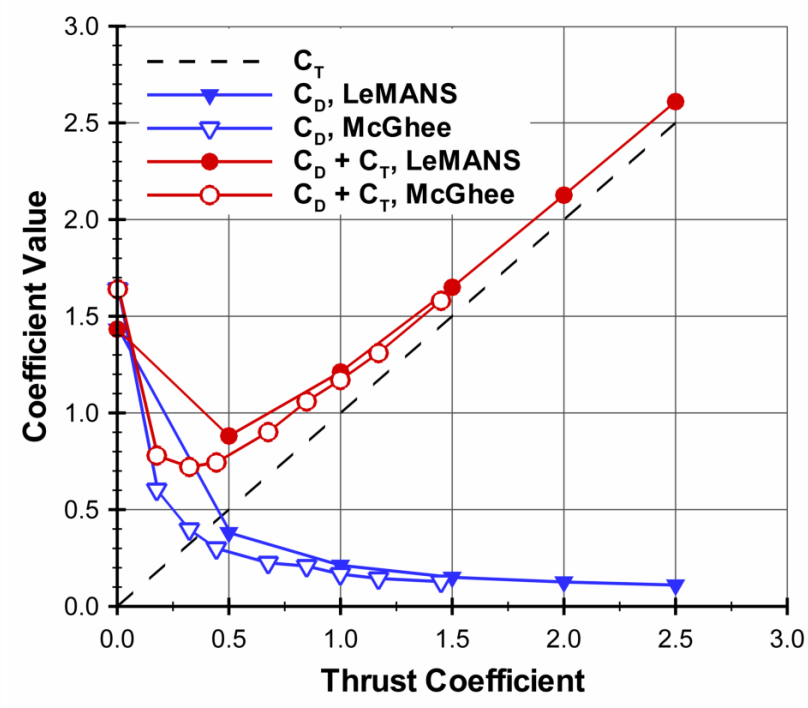

(b) Aerodynamic coefficients.

Figure 13. Comparison of bow shock standoff distance and aerodynamic coefficients between computational and experimental data.

\section{Conclusion}

The purpose of this study was to investigate the interactions of single-nozzle sonic PD jet on Mars entry aeroshells. The paper described numerical and experimental methods that were used to understand these complex flow interactions. Using the CFD code LeMANS, the effects on the flowfield, surface, and aerodynamic properties around an MSL-based aeroshell were evaluated for Mach 12 flow of iodine-seeded nitrogen gas. A sonic nozzle with an inlet-to-exit area ratio of 21 was placed at the center of the aeroshell forebody to supply a sonic PD jet. The boundary conditions for this jet were specified using a non-dimensional nozzle thrust coefficient in order to be able to compare the numerical results with experimental data from previous and ongoing work. The first part of this study focused on the flowfield effects of the PD jet. The results showed that as the thrust coefficient increases, the bow shock, jet shock, and interface region all move upstream, while the recirculation regions on either side of the PD jet move downstream towards the aeroshell shoulder and their size is reduced. The results also showed that the size of the PD jet increases and more jet species are transported to the wake region as the thrust coefficient increases. The second part of this study examined the effects of the PD jet on the surface properties of the aeroshell. The numerical 
results showed that the overall magnitudes of the pressure and shear stress decrease and approach a roughly constant value along the surface of the aeroshell as the thrust coefficient increases. In the third section of the study, the aerodynamic effects of the PD jet were investigated. It was found that the aerodynamic drag decreases and asymptotically nears a constant value of $8 \%$ of the PD jet off value as the thrust coefficient increases. The results also showed that the total axial force coefficient (i.e. the sum of the aerodynamic drag and thrust coefficients) does not reach a value greater than the drag coefficient value for the PD jet off case until a thrust coefficient of 1.25, where most of the contibution is from the PD thrust. In the last section of this study, comparisons between LeMANS and experiemental data showed good agreement in the bow shock profile and standoff distance as well as the aerodynamic coefficients.

\section{Future Work}

For future work, the interactions of single-nozzle supersonic and multiple-nozzle PD jets will be studied in order to understand the effects of the PD jet nozzle-exit Mach number and configuration on the flowfield, surface, and aerodynamic properties of Mars entry aeroshells. Using LeMANS, simulations of Mars entry capsules with PD jets in realistic conditions, such as Mars atmospheric conditions and reacting gas for the PD jets, will be undertaken to understand the aerothermodynamic effects of using a propulsive deceleration component to reduce the velocity of Mars aeroshells during atmospheric entry.

\section{Acknowledgments}

The authors gratefully acknowledge funding for this work through NASA Grant NNX08AH37A. The use of supercomputers at the University of Michigan (Center for Advanced Computing) and NASA (NASA Advanced Supercomputing Division) is essential to this work and is also greatly appreciated.

\section{References}

\footnotetext{
${ }^{1}$ Edquist, K. T., Dyakonov, A. A., Wright, M. J., and Tang, C. Y., "Aerothermodynamic Environments Definition for the Mars Science Laboratory Entry Capsule," AIA A Paper 2007-1206, January 2007.

${ }^{2}$ Korzun, A. M. and Braun, R. D., "Performance Characterization of Supersonic Retropropulsion for Application to High-Mass Mars Entry, Descent, and Landing," AIAA Paper 2009-5613, August 2009.

${ }^{3}$ Korzun, A. M., Braun, R. D., and Cruz, J. R., "Survey of Supersonic Retropropulsion Technology for Mars Entry, Descent, and Landing," Journal of Spacecraft and Rockets, Vol. 46, No. 5, September-October 2009, pp. 929-937.

${ }^{4} \mathrm{McDaniel}$, J. C., Glass, C. E., Staack, D., and Miller, C. G., "Experimental and Computational Comparisons of an Underexpanded Jet Flowfield," AIAA Paper 2002-0305, January 2002.

${ }^{5}$ Cecil, D. E. and McDaniel, J. C., "Planar Laser-Induced Iodine Fluorescence Measurements in Rarefied Hypersonic Flow," Rarefied Gas Dynamics: 24th International Symposium, Toronto, Canada, 2005, pp. 1325-1350.

${ }^{6}$ Reed, E. M., Codoni, J., McDaniel, J. C., Alkandry, H., and Boyd, I. D., "Investigation of the Interactions of Reaction Control Systems with Mars Science Laboratory Aeroshell," AIAA Paper 2010-1558, January 2010.

${ }^{7}$ Scalabrin, L. C. and Boyd, I. D., "Development of an Unstructured Navier-Stokes Solver for Hypersonic Nonequilibrium Aerothermodynamics," AIAA Paper 2005-5203, June 2005.

${ }^{8}$ Scalabrin, L. C. and Boyd, I. D., "Numerical Simulation of Weakly Ionized Hypersonic Flow for Reentry Configurations," AIAA Paper 2006-3773, June 2006.

${ }^{9}$ Scalabrin, L. C. and Boyd, I. D., "Numerical Simulations of the FIRE-II Convective and Radiative Heating Rates," AIAA Paper 2007-4044, June 2007.

${ }^{10}$ Holman, T. D. and Boyd, I. D., "Effects of Continuum Breakdown on the Surface Properties of a Hypersonic Sphere," Journal of Thermophysics and Heat Transfer, Vol. 23, No. 4, October-December 2009, pp. 660-673.

${ }^{11}$ Wilke, C. R., "A Viscosity Equation for Gas Mixtures," Journal of Chemical Physics, Vol. 18 No. 4, 1950, pp. 517-519.

${ }^{12}$ Blottner, F. G., Johnson, M., and Ellis, M., "Chemically Reacting Viscous Flow Program for Multi-Component Gas Mixtures," Tech. rep., SC-RR-70-754, Sandia Laboratories, Albuquerque, New Mexico, 1971.

${ }^{13}$ Vincenti, W. G. and Kruger, C. H., Introduction to Physical Gas Dynamics, Krieger Publishing Company, 2002.

${ }^{14}$ Karypis, G. and Kumar, V., "METIS: A Software Package for Partitioning Unstructured Graphs, Partitioning Meshes, and Computing Fill-Reducing Orderings of Sparse Matrices," University of Minnesota, 1998.

${ }^{15}$ Ashkenas, H. and Sherman, F. S., "The Structure and Utilization of Supersonic Free Jets in Low Density Wind Tunnels," Contract No. NAS7-100, ONR/AFOSR Contract Review, 1965.

${ }^{16}$ Alkandry, H., Boyd, I. D., Reed, E. M., and McDaniel, J. C., "Numerical Study of Hypersonic Wind Tunnel Experiments for Mars Entry Aeroshells," AIAA Paper 2009-3918, June 2009.

${ }^{17}$ McGhee, R. J., "Effects of a Retronozzle Located at the Apex of a 140 degree Blunt Cone at Mach Numbers of 3.00, 4.50, and 6.00," NASA Technical Note D-6002, January 1971.

${ }^{18}$ Pointwise Ver. 16 User's Manual. Pointwise 2010.
} 